\title{
Activity-Induced Polo-Like Kinase 2 Is Required for Homeostatic Plasticity of Hippocampal Neurons during Epileptiform Activity
}

\author{
Daniel P. Seeburg and Morgan Sheng \\ The Picower Institute for Learning and Memory, RIKEN-Massachusetts Institute of Technology Neuroscience Research Center, Howard Hughes Medical \\ Institute, Massachusetts Institute of Technology, Cambridge, Massachusetts 02139
}

\begin{abstract}
Homeostatic plasticity mechanisms stabilize the activity of a neuron or neuronal circuit during prolonged periods of increased network activity and have been proposed to function in the prevention of epilepsy. How homeostatic plasticity is achieved at the molecular level during hyperactivity states in general, and during epileptiform activity in particular, is unclear. Using organotypic hippocampal slice cultures as a model system, we found that the protein kinase Polo-like kinase 2 (Plk2) was induced during prolonged epileptiform activity and was required for the activity-dependent reduction in membrane excitability of pyramidal neurons. Disruption of Plk 2 function by dominant-negative or RNA interference not only blocked the downregulation of membrane excitability during epileptiform activity, but also unmasked a slow and progressive potentiation in synaptic strength that prevented the ability of the slice to undergo long-term potentiation. Thus, Plk2 function is required to prevent escalating potentiation and maintain synapses in a plastic state during epileptiform activity in hippocampal slice cultures.
\end{abstract}

Key words: Plk2; epilepsy; epileptiform; intrinsic membrane excitability; synaptic plasticity; homeostatic plasticity; organotypic hippocampal slice culture

\section{Introduction}

Homeostatic plasticity stabilizes neural networks in the face of perturbations like changes in neuronal size during development or changes in the strength and number of synapses (Davis, 2006; Turrigiano, 2007). As such, it has emerged as an important complement to Hebbian plasticity mechanisms, like NMDA receptor-dependent long-term potentiation (LTP) or depression, that on their own would tend to destabilize neural networks by causing runaway excitation or quiescence. How homeostatic plasticity is achieved at the molecular level, however, has remained largely unclear, although there has been some progress in understanding the homeostatic response to decreased network activity in dissociated neuron cultures (Rutherford et al., 1998; Shepherd et al., 2006; Stellwagen and Malenka, 2006).

Polo-like kinases (Plks) are a family of serine/threonine kinases primarily known for their role in regulation of cell cycle progression (van de Weerdt and Medema, 2006). Recently, we identified Plk2 as part of a novel molecular mechanism required in postmitotic hippocampal cultures for downward scaling of synaptic strength after chronic ( $48 \mathrm{~h}$ ) elevation of activity (See-

\footnotetext{
Received Jan. 4, 2008; accepted May 20, 2008.

M.S. is an investigator of the Howard Hughes Medical Institute. We thank Chinfei Chen and Chris Nelson for careful reading of this manuscript.

Correspondence should be addressed to Morgan Sheng, The Picower Institute for Learning and Memory, Massachusetts Institute of Technology, 77 Massachusetts Avenue (46-4303), Cambridge, MA 02139. E-mail: msheng@mit.edu.

DOI:10.1523/JNEUROSCI.1853-08.2008

Copyright $\odot 2008$ Society for Neuroscience $\quad$ 0270-6474/08/286583-09\$15.00/0
}

burg et al., 2008). Activity-induced Plk2 caused weakening of synapses through phosphorylation and degradation of SPAR, a postsynaptic Rap GAP (GTPase-activating protein) and scaffolding protein (Seeburg et al., 2008). Activity-induced Plk2 also causes loss of mature spines and synapses as well as loss of clusters of postsynaptic density-95 (PSD-95), a component of the PSD of excitatory synapses (Pak and Sheng, 2003; Sheng and Hoogenraad, 2007). Thus, Plk2 seems to participate critically in the classical negative feedback response (synaptic scaling) to elevated activity, which is conventionally studied in dissociated neuron cultures. However, little is known about the role or molecular mechanisms of homeostatic plasticity in response to elevated activity in a more intact system or in vivo.

A clinically relevant form of elevated neuronal activity is that associated with epileptic seizures, which are caused by paroxysmal hypersynchronous bursts of activity in networks of neurons in the CNS. Such activity can be modeled in organotypic hippocampal slice cultures, which retain the general cytoarchitecture and connectivity of the intact brain structure (Gahwiler et al., 1997; Holopainen, 2005), and show robust epileptiform (highly synchronous bursting) activity as well as many of the pathophysiological hallmarks of seizures after treatment with convulsants (Thompson et al., 1996; Bausch and McNamara, 2004; Zha et al., 2005). Homeostatic plasticity mechanisms are believed to provide endogenous protection against recurring forms of such epileptiform activity, although few studies have tested this idea experimentally. Interestingly, expression of Plk 2 is induced over the course of a few hours in rat brain in vivo after seizures induced 
pharmacologically (Kauselmann et al., 1999) or by electroconvulsive shock treatment (Newton et al., 2003).

In this study, we report that Plk2 is induced in organotypic hippocampal slice cultures during epileptiform activity and prevents activity-dependent potentiation of synaptic strength. When Plk2 function was blocked during epileptiform activity, synaptic strength escalated, precluding the slice from undergoing further potentiation through LTP. Thus, Plk2-dependent plasticity mechanisms stabilize neuronal activity and keep synapses plastic during epileptiform activity in slice culture.

\section{Materials and Methods}

DNA constructs, drugs, and antibodies. The Plk2 RNA interference (RNAi) construct (with small interfering RNA target sequence $5^{\prime}$ GCATAAGAGAAGCAAGATA-3') in pSUPER (Brummelkamp et al., 2002) and green fluorescent protein (GFP)-polo box domain (PBD) have been described previously (Seeburg et al., 2008). The RNAi construct against firefly luciferase (5'-CCGCCTGAAGTCTCTGATTAA-3') in pENTR-Mir U6 vector was a gift from Wade Harper (Harvard Medical School, Boston, MA). Drugs used in this study were purchased from Sigma. Polyclonal Plk2 antibody has been described previously (Pak and Sheng, 2003). Polyclonal GFP antibody (MBL) and monoclonal $\alpha$-tubulin (B-5-1-2) antibody (Sigma) were purchased.

Hippocampal slice culture, transfection, and immunostaining. Organotypic hippocampal slice cultures were prepared from postnatal 8-d-old rats as described previously (Stoppini et al., 1991; Sala et al., 2003). Briefly, after anesthesia and decapitation, brains were removed and placed into an ice-cold dissection buffer containing the following (in mM): 238 sucrose, $2.5 \mathrm{KCl}, 26 \mathrm{NaHCO}_{3}, 1 \mathrm{NaH}_{2} \mathrm{PO}_{4}, 11$ glucose, 5 $\mathrm{MgCl}_{2}$, and $1 \mathrm{CaCl}_{2}$. The hippocampi were dissected out, cut into 350$\mu \mathrm{m}$-thick slices using a McIlwain tissue chopper, and plated onto tissue plate inserts (Millipore) placed over MEM-based (Cellgro) culture medium supplemented with horse serum (Invitrogen), insulin $(2 \mu \mathrm{g} / \mathrm{ml})$, and ascorbic acid $(0.0012 \%)$, containing the following (in $\mathrm{mm}$ ): 26 D-glucose, $5.8 \mathrm{NaHCO}_{3}, 30 \mathrm{HEPES}, 2 \mathrm{CaCl}_{2}$, and $2 \mathrm{MgCl}_{2}$. Slices were incubated in $5 \% \mathrm{CO}_{2}$ at $35^{\circ} \mathrm{C}$ and transfected on days in vitro (DIV) 5-7 (GFP-PBD) or DIV 3-5 (Plk2 RNAi) using a biolistic gene gun (Bio$\mathrm{Rad})$. Gold particles $(1.6 \mu \mathrm{m} ; \sim 0.3 \mathrm{mg}$ per cartridge) were coated with DNA plasmids: GFP-PBD $(100 \mu \mathrm{g})$, or Plk2 RNAi $(90 \mu \mathrm{g})$ together with a GFP-expression vector $(10 \mu \mathrm{g})$ for visualization of transfected cells. Recordings and/or fixation of slices occurred on DIV 6-8. Slices were fixed in $4 \%$ paraformaldehyde, $4 \%$ sucrose in PBS overnight, then cryoprotected in 30\% sucrose in $0.1 \mathrm{~m}$ phosphate buffer, $\mathrm{pH} 7.4$, for $2 \mathrm{~h}$ at room temperature (RT), snap frozen on dry ice, thawed in PBS, and stained with GFP antibodies in GDB buffer ( $0.1 \%$ gelatin, $0.3 \%$ TX- 100 , $450 \mathrm{~mm} \mathrm{NaCl}$, and $32 \% 0.1 \mathrm{~m}$ phosphate buffer, $\mathrm{pH} 7.4$ ).

Sindbis virus preparation and slice infection. Plk2 was cloned into a modified pSinRep5 vector containing GFP preceded by an IRES2 internal ribosomal entry site. Sindbis virus was prepared according to the manufacturer's guidelines (Invitrogen). Briefly, DNA templates were transcribed using an in vitro transcription kit (Ambion), and then electroporated into BHK cells. Twenty-four to 36 hours after transfection, the supernatant was harvested and used for organotypic hippocampal slice infection by directly injecting virus into the CAl region of the slice using a picospritzer II (Parker Instrumentation). Recordings were performed $\sim 24 \mathrm{~h}$ after infection under visual guidance of the GFP signal.

Electrophysiology. Electrophysiological recordings were performed from organotypic slice cultures as described previously (Sala et al., 2003). Recordings were performed at $1 \mathrm{~d}$ (GFP-PBD overexpression) or 3-4 d (Plk2 RNAi) after transfection in solution containing the following (in mM): $119 \mathrm{NaCl}, 2.5$ $\mathrm{KCl}, 4 \mathrm{CaCl}_{2}, 4 \mathrm{MgCl}_{2}, 26 \mathrm{NaHCO}_{3}, 1 \mathrm{NaH}_{2} \mathrm{PO}_{4}, 11$ glucose, 0.1 picrotoxin, and 0.002 2-chloroadenosine, gassed with $5 \% \mathrm{CO}_{2} / 95 \% \mathrm{O}_{2}$ at $\mathrm{pH}$ 7.4. For I-clamp and miniature EPSC (mEPSC) recordings, 2-chloroadenosine was omitted, the $\mathrm{CaCl}_{2}$ and $\mathrm{MgCl}_{2}$ concentrations were 2.8 and $2 \mathrm{~mm}$, respectively, and recordings were performed at $30-34^{\circ} \mathrm{C}$. TTX $(1 \mu \mathrm{M})$ was added during mini-EPSC recordings. LTP and evoked EPSC (eEPSC) recordings were performed at RT. For I-clamp recordings, excitatory synaptic currents were blocked with APV $(50 \mu \mathrm{M})$ and 2,3-dihydroxy-6-nitro-7-sulfonyl- benzo[f] quinoxaline (NBQX; $10 \mu \mathrm{M}$ ); no picrotoxin was added to the perfusion. Whole-cell recordings were made simultaneously from a pair of CA1 pyramidal neurons, one transfected and one untransfected. For eEPSCs, presynaptic fibers were stimulated at $0.2 \mathrm{~Hz}$ with a bipolar electrode with single voltage pulse ( $200 \mu \mathrm{s}$, up to $10 \mathrm{~V}$ ) placed in stratum radiatum $\sim 200$ $\mu \mathrm{m}$ from recorded cells. LTP was induced by pairing $2 \mathrm{~Hz}$ stimulation with depolarization of the postsynaptic cell to $0 \mathrm{mV}$ for $100 \mathrm{~s}$.

For voltage-clamp recordings, pipettes $(2-4 \mathrm{M} \Omega)$ were filled with an internal solution containing the following (in $\mathrm{mM}$ ): 115 cesium methanesulfonate, $20 \mathrm{CsCl}$, 10 HEPES, $2.5 \mathrm{MgCl}_{2}$, 4 ATP disodium salt, $0.4 \mathrm{GTP}$ trisodium salt, 10 sodium phosphocreatine, and 0.6 EGTA, at pH 7.25. For current-clamp recordings, the internal solution contained the following (in $\mathrm{mm}$ ): 100 potassium methanesulfonate, $20 \mathrm{KCl}, 10$ HEPES, 4 ATP magnesium salt, 10 sodium phosphocreatine, and 0.03 GTP trisodium salt, $\mathrm{pH} 7.3$.

Current-clamp recordings to measure intrinsic excitability were performed as described by Karmarkar and Buonomano (2006). Briefly, recordings were considered acceptable if the cells showed overshooting action potentials (APs) and their resting membrane potential did not vary by $>5 \mathrm{mV}$. Additionally, the input resistance was required to be $>80$ $\mathrm{M} \Omega$ and to not change by $>15 \%$ during the course of the recording. Intrinsic excitability of an individual cell was measured as the number of spikes in response to a series of six fixed $250 \mathrm{~ms}$ current injection steps $(0.05,0.1,0.15,0.2,0.25$, and $0.3 \mathrm{nA})$. The number of spikes was averaged over three sweeps for each current intensity. Current was injected to keep cells at a baseline membrane potential of $-60 \mathrm{mV}$ between current injection steps.

Whole-cell current and voltage-clamp recordings were made using a Multiclamp 700A amplifier (Molecular Devices). Voltage and current signals were filtered at $2 \mathrm{kHz}$ and digitized at $10 \mathrm{kHz}$ with a Digidata 1322A (Molecular Devices). Analysis of recordings was performed using Clampfit software (Molecular Devices).

Imaging and quantitation. Microscope images were acquired using an LSM510 confocal system with a water-immersion $63 \times$ objective (numerical aperture 1.2; Zeiss). Confocal $Z$-series image stacks encompassing entire dendrite segments were analyzed using MetaMorph software (Molecular Devices). For quantitation of spine size and density in slice cultures, measurements were made from the middle third $(\sim 70-100 \mu \mathrm{m}$ in length) of the primary apical dendritic branch and averaged for each neuron.

Statistical analysis. Statistical significance was evaluated by the MannWhitney test, unless otherwise noted. Statistical significance was set at $p<0.05$.

\section{Results}

Transient potentiation and induction of Plk2 protein during epileptiform activity in organotypic hippocampal slices

Pharmacological blockade of $\mathrm{GABA}_{\mathrm{A}}$ receptor-mediated inhibition in hippocampal slices is a commonly used model for studying mechanisms of epilepsy (Schwartzkroin and Prince, 1978; Traub and Wong, 1982; Drakew et al., 1996; Thompson et al., 1996; Karnup and Stelzer, 2001). Under control conditions, organotypic hippocampal cultures at DIV 5-7 displayed little spontaneous spiking activity in CA1 or CA3 pyramidal cells (Fig. $1 \mathrm{~A}$ ). However, after treatment with picrotoxin (PTX; $100 \mu \mathrm{M}$ ), a $\mathrm{GABA}_{\mathrm{A}}$ receptor antagonist and convulsant, we observed synchronous bursting discharges in CA1 and CA3 pyramidal cells with burst durations ranging from $\sim 0.5 \mathrm{~s}$ to several seconds in length (Fig. $1 B$; supplemental Figure $\mathrm{S} 1$, available at www. jneurosci.org as supplemental material). Bursting appeared rapidly after application of PTX and occurred regularly about every $30-60$ s. Over the course of $18 \mathrm{~h}$ of PTX treatment, the average burst length dropped by $\sim 60 \%$ from (mean \pm SEM) $4.07 \pm$ $0.52 \mathrm{~s}$ to $1.71 \pm 0.26 \mathrm{~s}$, whereas the frequency of bursts increased from $0.61 \pm 0.1$ bursts per minute to $1.71 \pm 0.26$ bursts per minute (data not shown).

Plk2 protein levels rose steadily over the course of $\sim 6-12 \mathrm{~h}$ in 


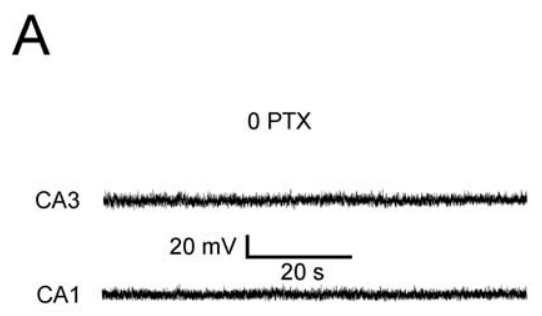

B

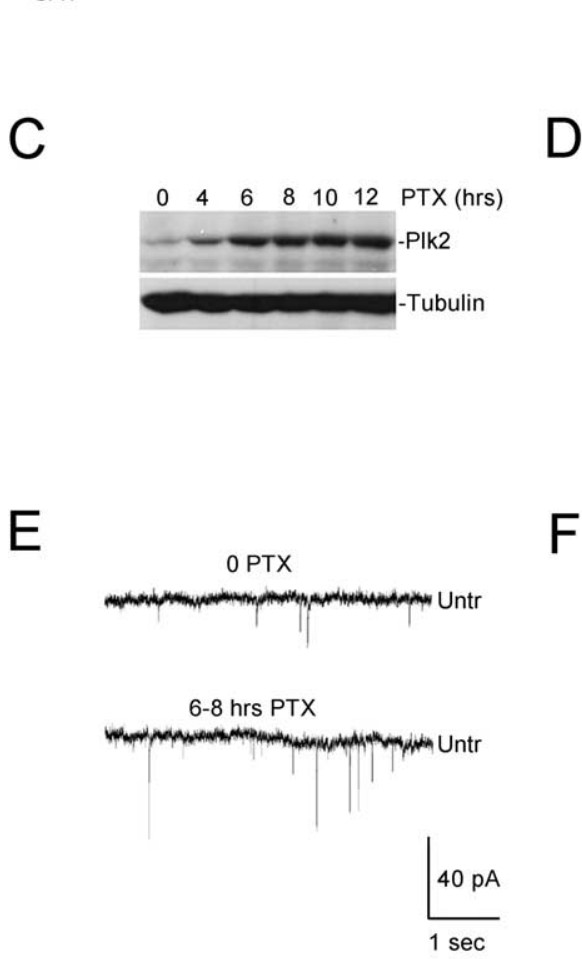

$\mathrm{D}$
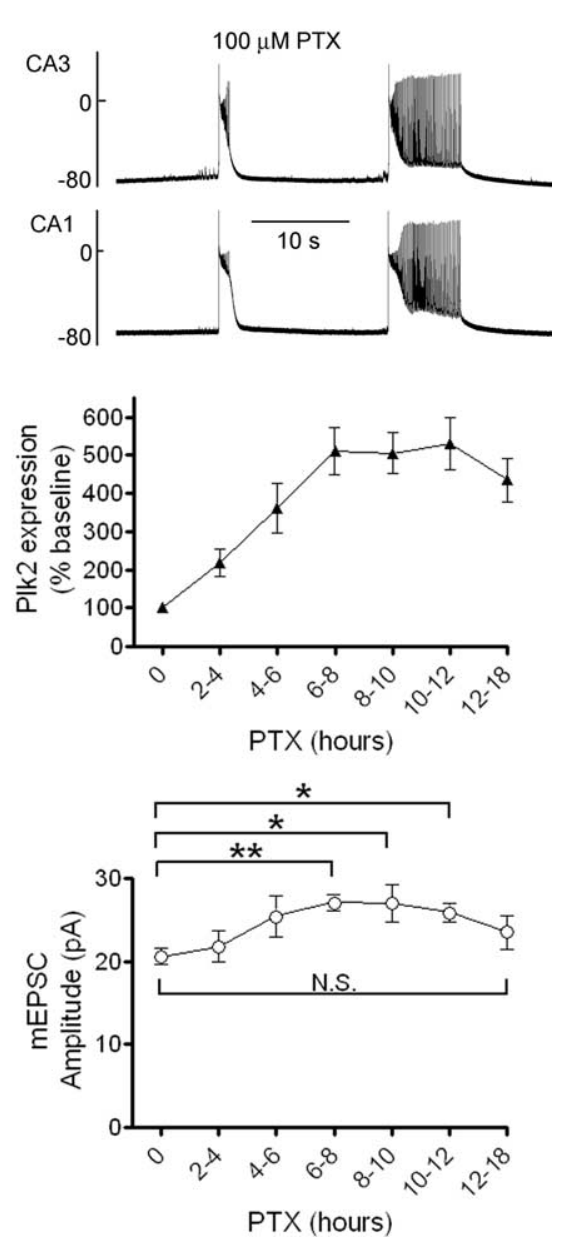

Figure 1. Effect of epileptiform activity on Plk2 expression and synaptic strength in organotypic hippocampal cultures. $\boldsymbol{A}$, No bursting activity is seen in cultured hippocampal slices (DIV 6-8) under basal conditions. $\boldsymbol{B}$, PTX (100 $\mu \mathrm{m}$ ) elicits synchronous bursting (epileptiform) activity in CA1 and CA3. C, D, Induction of Plk2 protein expression during epileptiform activity. C, Hippocampal slice cultures (DIV 6-8) were treated with PTX for indicated times (hours), and then immunoblotted for PIk2 and tubulin. D, Quantification of Plk2 protein levels was performed by densitometry (mean \pm SEM), normalized to vehicle-treated controls. $n=7-8$ experiments for each time point. $\boldsymbol{E}, \boldsymbol{F}$, Time course of change in mEPSC amplitude during PTX treatment in hippocampal slice culture. $\boldsymbol{E}$, Representative mEPSC traces from untransfected (Untr) CA1 pyramidal cells in hippocampal slice cultures (DIV 6 - 8) are shown with or without $6-8 \mathrm{~h}$ of PTX treatment, as indicated. $\boldsymbol{F}$, mEPSC amplitudes (mean \pm SEM) were plotted against duration of PTX treatment. $n=8-10$ cells for all time points. ${ }^{*} p<0.05$; ${ }^{* *} p<0.001$.

hippocampal slice cultures undergoing PTX-induced epileptiform activity (Fig. 1C, quantified in D). Prolonged (overnight) synchronized bursting activity in these cultures has been reported to lead to modest potentiation $(\sim 25 \%)$ of AMPA receptor (AMPAR)-mediated mEPSCs recoded from CA1 pyramidal cells (Abegg et al., 2004). Examining the detailed time course of this bursting-induced potentiation, we found a steady and significant increase in mEPSC amplitude in CA1 pyramidal cells over the first $6-8 \mathrm{~h}$ of PTX treatment to $\sim 132 \%$ of baseline (baseline: $20.6 \pm 1.03 \mathrm{pA}, n=12 ; 6-8$ h PTX: $27.1 \pm 1.01 \mathrm{pA}, n=8 ; p<$ 0.01 ) (Fig. $1 E$, quantified in $F$ ). After that, however, mEPSC amplitudes failed to increase further over the next $6-10 \mathrm{~h}$, despite the continued presence of PTX and epileptiform bursting. In fact, at $12-18 \mathrm{~h}$ of PTX treatment, mEPSC amplitude was no longer significantly different from baseline $(114 \pm 9.9 \% ; n=10 ; p=$ 0.41) (Fig. $1 F)$. A similar lack of difference in mEPSC amplitude between basal conditions and $12-18 \mathrm{~h}$ of PTX treatment is also seen in subsequent experiments (see below). The temporal profile of mEPSC amplitude raises the possibility that homeostatic plasticity mechanisms are being activated during $\sim 6-18 \mathrm{~h}$ of synchronized bursting activity to maintain synaptic strength close to the normal range. The time of maximal mEPSC amplitude coincided approximately with the peak of endogenous Plk2 protein levels induced by PTX (at $\sim 6-10$ h) (Fig. 1, compare $D, F$ ).

\section{Plk2 prevents potentiation of synaptic strength during epileptiform activity} We hypothesized that the induced Plk2 plays a role in terminating the rise in mEPSC amplitude during epileptiform activity. To test this idea, we transfected a Plk2 dominant-negative construct, consisting of the Plk2 C-terminal PBD fused to GFP (GFP-PBD), into individual CA1 pyramidal cells. The PBD mediates subcellular targeting of Plks (Lowery et al., 2004), and PBDs of Plk1 and Plk3 have been used as dominant-negative constructs against endogenous Plk1 and Plk3, respectively (Song et al., 2000; Song and Lee, 2001; Seong et al., 2002; Jiang et al., 2006). The GFP-PBD construct of Plk2 works effectively as a dominant-negative to block conventional synaptic scaling in dissociated hippocampal neuron cultures treated chronically with PTX (Seeburg et al., 2008). We found that in contrast to neighboring untransfected cells in the same slice, neurons expressing GFP-PBD underwent progressive strengthening of mEPSCs that failed to plateau or decline during $18 \mathrm{~h}$ of PTX treatment, reaching $187 \%$ of baseline (baseline: $19.15 \pm 0.98 \mathrm{pA}, n=11 ; 12-18 \mathrm{~h}$ PTX: $35.89 \pm 3$ pA, $n=10 ; p<0.001)$ (Fig. $2 A$, quantified in Fig. $2 B$, where the untransfected data are replotted from Fig. $1 F$ for comparison). As a result, the mean mEPSC amplitude after 12-18 h of PTX treatment was significantly greater in GFPPBD-transfected cells than in untransfected controls (153 $\pm 13 \%$ of control; $p<0.01$, two-way ANOVA with posttest) (Fig. 2 B). Compared with untransfected cells, the frequency of mEPSCs showed a trend toward higher frequencies in cells transfected with $\mathrm{Plk} 2$ dominant-negative GFP-PBD (supplemental Fig. S2, available at www.jneurosci.org as supplemental material), although this did not reach statistical significance.

As an alternative way to disrupt Plk2 function, we transfected into individual CA1 neurons an RNAi construct against Plk2 to suppress expression of endogenous Plk2 (Seeburg et al., 2008). Cells transfected with Plk2-RNAi construct (plus GFP construct for visualization of transfected cells) 2-3 d before PTX treatment showed an elevated mEPSC amplitude after 12-18 h of PTX treatment $(150 \pm 10 \%$ of baseline; $n=7 ; p<0.01)$ that was $\sim 30 \%$ greater than in neighboring untransfected cells in the same slice $(n=7 ; p<0.05)$ (Fig. $2 C$, quantified in $D)$. In contrast, a control RNAi construct expressing a small hairpin RNA against firefly luciferase (FF RNAi) (together with a GFP construct for visualization of transfected cells) had no effect on mEPSC amplitude 
relative to untransfected cells after $12-18 \mathrm{~h}$ of PTX treatment $(97 \pm 8 \%$ of control; $p=$ 0.9 ) (Fig. 2E,F). Neither GFP-PBD nor Plk2 RNAi caused a change in mEPSC amplitude relative to untransfected cells under basal (untreated) conditions (Fig. $2 B, D)$, which suggests that these constructs do not have nonspecific toxic effects, and is in keeping with the fact that Plk2 was expressed at very low levels in unstimulated slice cultures (Fig. 1C). The RNAi results corroborate the dominantnegative findings, indicating that $\mathrm{Plk} 2$ is required to prevent the escalation of synaptic strength during prolonged $(>8 \mathrm{~h})$ epileptiform activity. (For the purpose of this study, we henceforth use the term "epileptiform potentiation" to refer specifically to the progressive increase in synaptic strength induced by $12-24 \mathrm{~h}$ PTX in neurons with disrupted Plk2 function.)

When PTX treatment was extended past $24 \mathrm{~h}$ to 48 and $72 \mathrm{~h}$, endogenous Plk2 protein levels in cultured hippocampal slices dropped back down to baseline levels, despite the continued presence of PTX (Fig. 3A). After $48 \mathrm{~h}$ of PTX, mEPSC amplitudes became significantly strengthened again relative to untreated control cultures (Fig. $3 B, C$, quantified in $D$ ). Overexpression of Plk2 in CA1 neurons by Sindbis viral infection prevented the strengthening of mEPSC amplitude at these late time points, whereas overexpression of GFP alone by Sindbis virus infection did not (Fig. 3B-D). Thus not only is induction of Plk2 necessary, but overexpression of Plk2 is sufficient, to prevent potentiation of synaptic strength during PTX-induced epileptiform activity.

\section{Pharmacological characterization of epileptiform potentiation}

What is the mechanism of the "epileptiform potentiation" unmasked by dominant-negative or RNAi disruption of Plk2 function (Fig. 2 $B, D$ )? Epileptiform activity induced by PTX treatment in hippocampal slices consists of synchronous and thus highly correlated presynaptic and postsynaptic activity between CA1 and CA3 pyramidal cells (Fig. $1 B$ ), a condition expected to be conducive toward induction of LTP. However, coapplication of NMDA receptor (NMDAR) antagonist APV (100 $\mu \mathrm{M})$ with PTX $(100 \mu \mathrm{M})$ did not prevent epileptiform potentiation in cells transfected with GFP-PBD (155 $\pm 12 \%$ of baseline; $n=8 ; p<0.01$ ) (Fig. $4 A$ ), indicating that this pathological form of potentiation does not require NMDAR activity. Interestingly, in untransfected (Plk2-intact) cells that normally maintain synaptic strength close to baseline after $12-18 \mathrm{~h}$ of PTX treatment, APV blockade of NMDARs during synchronized bursting activity actually caused mEPSC amplitude to rise to $152 \pm 9 \%(n=8)$ of baseline (cf. $116 \pm 7 \%$ with PTX alone; $n=5$; PTX + APV significantly different from PTX alone, $p<0.05$ ) (Fig. $4 A$ ). These findings suggest that the homeostatic mechanisms preventing
B
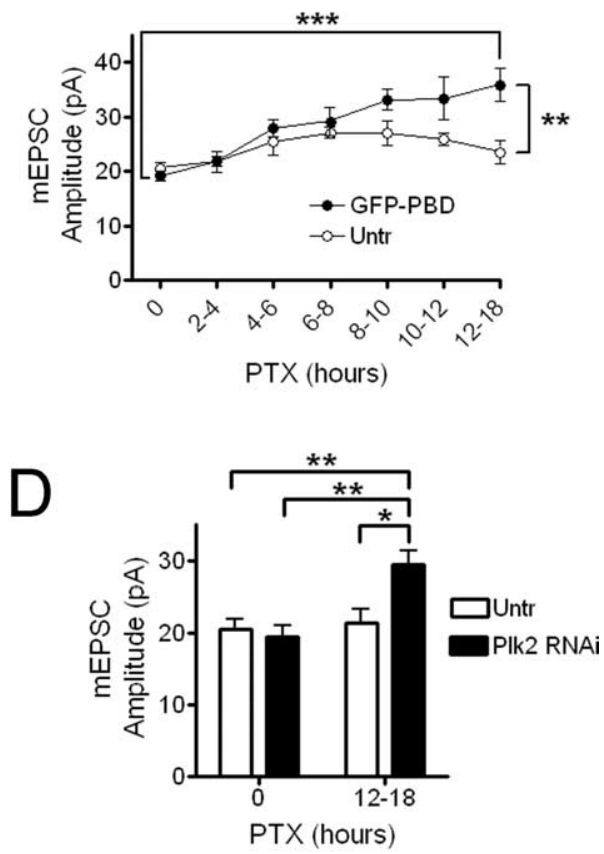

E

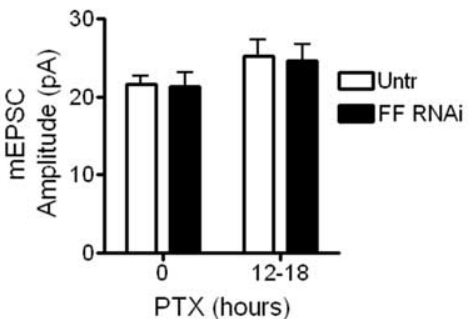

PTX (hours)

Figure 2. Plk2 is required to prevent synaptic potentiation during epileptiform activity in hippocampal slice cultures. $\boldsymbol{A}, \boldsymbol{B}$, Time course of change in mEPSC amplitude during PTX treatment in neurons $24 \mathrm{~h}$ after transfection with GFP-PBD. $\boldsymbol{A}$, Represenindicated. $\boldsymbol{B}$, Quantified mEPSC amplitudes (mean \pm SEM) were plotted against duration of PTX treatment for GFP-PBDtransfected and untransfected neurons (untransfected data replotted from Fig. $1 F$ for comparison). $n=7-11$ cells for all time amplitude after 12-18 h of PTX treatment. Left, Representative mEPSC traces are shown from untransfected CA1 pyramidal cells versus cells transfected with RNAi construct against Plk2 (Plk2 RNAi; $\boldsymbol{C}$ ) or FF RNAi $(\boldsymbol{E})$. Both RNAi constructs were cotransfected amplitudes (mean \pm SEM) from cells transfected with RNAi constructs and treated with PTX or not, as indicated $(\boldsymbol{D}, \boldsymbol{F}) . n=6-7$ cells for each condition. ${ }^{*} p<0.05 ;{ }^{* *} p<0.01$.

epileptiform potentiation depend on NMDAR function as well as Plk2. It is worth recalling, however, that induction of Plk2 itself requires calcium influx through either NMDARs or voltagegated calcium channels; thus this NMDAR requirement could reflect the mechanism of induction of Plk2 (Pak and Sheng, 2003).

We next tested whether CaMKII, a protein kinase important for the induction of LTP (Malinow et al., 1989; Silva et al., 1992), is required for epileptiform potentiation. Coapplication of $\mathrm{KN}$ 93, a CaMKII inhibitor, with PTX had no effect on potentiation of synapses in GFP-PBD-transfected cells ( $159 \pm 7 \%$ of baseline; $n=6 ; p<0.01$ ), nor did it interfere with homeostatic mechanisms in untransfected cells $(94.7 \pm 3.5 \%$ of PTX alone; $n=5$; $p=0.8$ ) (Fig. 4A). Thus, epileptiform potentiation is inhibited by NMDARs and Plk2, and does not depend on CaMKII.

To measure the AMPA/NMDA ratio during epileptiform potentiation, we recorded simultaneously from a pair of neighbor- 
A
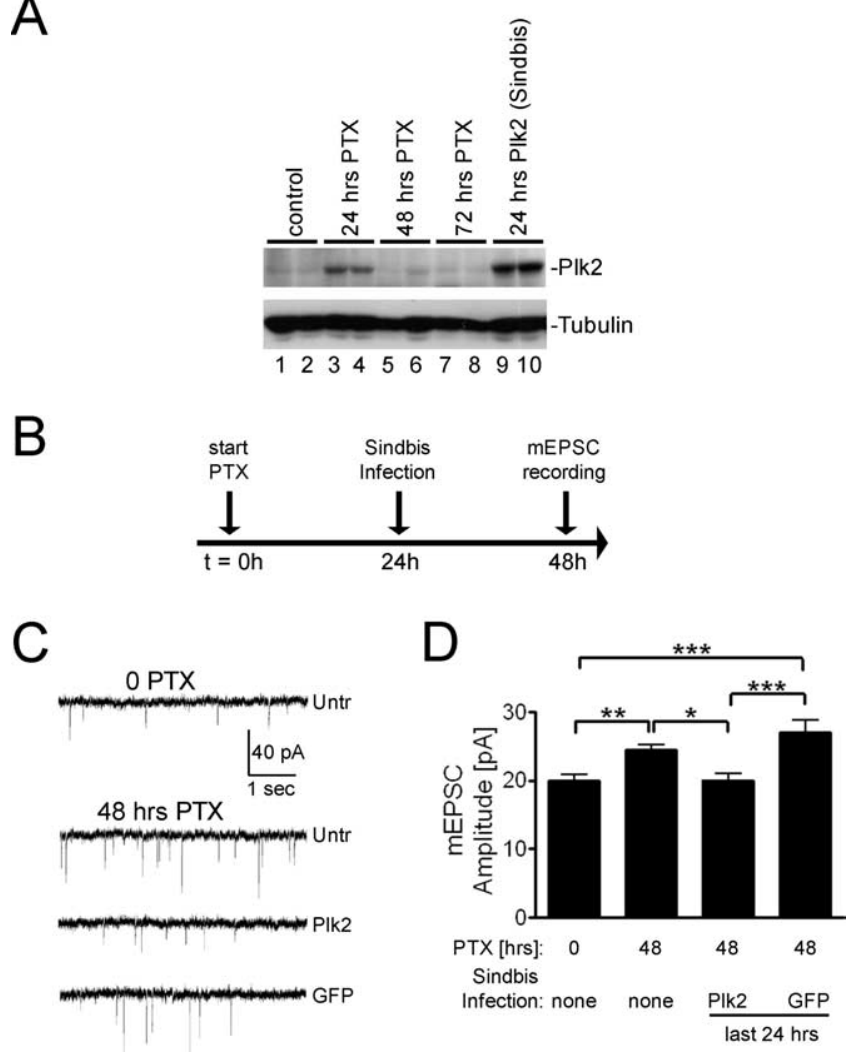

Figure 3. Plk2 dampens mEPSC amplitude in PTX-treated neurons. $\boldsymbol{A}$, Immunoblot showing Plk2 expression during extended PTX treatment (24-72 h). Hippocampal slice cultures (DIV $6-8)$ were treated with PTX $(100 \mu \mathrm{m})$ for indicated times and immunoblotted for PIk2 and tubulin. Slices not treated with PTX were infected with Sindbis virus vector expressing wild-type Plk2 for $\sim 24 \mathrm{~h}$ in lanes 9 and 10 . $\boldsymbol{B}-\boldsymbol{D}, 0$ verexpression of wild-type PIk2 reduces $m E P S C$ amplitude in PTX-treated slices. The time line of the experiment is schematized in $\boldsymbol{B}$. Hippocampal slice cultures were either untreated or treated with PTX for $48 \mathrm{~h}$; Sindbis viral infection was performed $\sim 24 \mathrm{~h}$ before mEPSCs were recorded. $\boldsymbol{C}$, Representative mEPSC traces from uninfected CA1 pyramidal cells and cells infected with Sindbis virus expressing PIk2 + GFP (PIk2) or GFP alone (GFP), with or without $48 \mathrm{~h}$ of PTX, as indicated. $D$, Quantitation of $m E P S C$ amplitudes (mean \pm SEM) recorded from CA1 pyramidal cells uninfected or infected with Sindbis virus expressing PIk2 + GFP (Plk2) or GFP alone (GFP), with or without treatment with $48 \mathrm{~h}$ of PTX, as indicated. $n=21$ cells ( 0 h PTX, uninfected); $n=27$ cells (48 h PTX, uninfected); $n=18$ cells (48 h PTX, Plk2 infection); $n=12$ cells (48 h PTX, GFP infection). ${ }^{*} p<0.05,{ }^{* *} p<0.01,{ }^{* * *} p<0.001$, one-way ANOVA. Untr, Untransfected.

ing GFP-PBD-transfected and untransfected cells in the CA1 region of hippocampal slice cultures, while eliciting eEPSCs by stimulating Schaffer collateral inputs. In untransfected cells, 12-18 h of PTX treatment caused a modest but significant increase in the AMPAR-to-NMDAR current ratio [149 $\pm 16 \%$ of untreated controls; $n=8$ ( 0 and 12-18 h PTX); $p<0.05$ ] (Fig. $4 B$, quantified in $C$ ). In cells transfected with Plk2 dominantnegative construct GFP-PBD, a much larger increase in the AMPA/NMDA ratio was observed after the same PTX treatment $[241 \pm 33 \%$ of untreated controls; $n=8$ ( 0 and 12-18 h PTX); $p<0.01$ ] (Fig. $4 B, C$ ). As a result, GFP-PBD-overexpressing neurons showed more than twofold higher AMPA/NMDA ratio than untransfected cells in the same PTX-stimulated slice (216 $\pm 29 \%$; $n=8 ; p<0.01$ ) (Fig. 4C). These results show that epileptiform potentiation is associated with an increase in the AMPA/NMDA EPSC ratio, and that Plk2 functions to dampen both the potentiation and the increased AMPA/NMDA ratio.

\section{Morphological correlates of epileptiform potentiation}

Synaptic potentiation is associated with enlargement of spine heads (Matsuzaki et al., 2004; Okamoto et al., 2004; Kopec et al., 2006). To test whether epileptiform potentiation involves changes in spine size, we transfected the Plk2 dominant interfering construct GFP-PBD into CA1 neurons of organotypic hippocampal cultures at DIV 5-7 and analyzed the effects on spine size and density $\sim 24 \mathrm{~h}$ after transfection. Under basal conditions (without PTX treatment), GFP-PBD had no effect on spine density or spine size (107 $\pm 7 \%$ of GFP spine density, $p=0.6$; $96 \pm$ $4 \%$ of GFP spine length, $p=0.6 ; 105 \pm 3 \%$ of GFP spine width, $p=0.3$ ) (Fig. $4 D-G$ ), consistent with low levels of Plk2 expression in unstimulated slices (Fig. 1C). In contrast, $12-18 \mathrm{~h}$ of PTX treatment resulted in significantly larger and more numerous spines in GFP-PBD-transfected neurons compared with untreated conditions $(142 \pm 4 \%$ of basal spine width, $p<0.001$; $123 \pm 6 \%$ of basal spine density, $p<0.05$ ), whereas spine length was unchanged (108 $\pm 2 \%$ of basal spine length; $p=0.08)$. A small increase in spine width was also seen in control GFPtransfected cells treated with PTX $(113 \pm 3 \%$ of basal spine width; $p<0.05)$, although this increase was significantly smaller than that seen in GFP-PBD-transfected neurons $(p<0.001)$ (Fig. $4 G$ ). The potentiation in mini-EPSC amplitude during epileptiform activity is thus correlated with an increase in spine size in cells with blocked Plk2 function. One might expect an increase in mini frequency with the increase in spine density, but as noted above, the elevation of mEPSC frequency did not reach statistical significance (supplemental Fig. S2, available at www.jneurosci. org as supplemental material).

\section{Epileptiform potentiation blocks further potentiation by LTP} We also examined the effects of dominant-negative Plk2 disruption on eEPSC amplitude. In accord with our mEPSC recordings, overexpression of GFP-PBD for $\sim 24 \mathrm{~h}$ had no effect on eEPSC amplitudes relative to neighboring untransfected cells in basal conditions (no PTX) (Fig. 5A). However, cells transfected with GFP-PBD showed significantly enhanced eEPSC amplitude after prolonged epileptiform activity (12-24 h PTX) (Fig. 5B), again consistent with our mEPSC recordings and suggesting disruption of normal homeostatic mechanisms (Fig. 2B).

What are the physiological consequences of the unchecked epileptiform potentiation that occurs when Plk2 function is blocked? Under basal conditions, cells transfected with GFPPBD showed robust LTP in response to a pairing protocol, similar in magnitude to neighboring untransfected cells in the same slice that were recorded simultaneously (Fig. 5C). After 12-24 h of PTX treatment, however, LTP could no longer be induced in CA1 neurons transfected with GFP-PBD (Fig. 5D). Importantly, neighboring untransfected cells in the same slice retained their ability to undergo LTP. These results indicate that epileptiform potentiation prevents further potentiation by LTP, perhaps by occlusion or saturation, and that normally, Plk2 functions to prevent epileptiform potentiation and to allow synapses to remain plastic in the face of chronic hyperactivity.

\section{Plk2 reduces neuronal excitability during increased network activity}

Prolonged increases in network activity in organotypic hippocampal cultures are associated with a compensatory reduction in neuronal membrane excitability (Karmarkar and Buonomano, 2006). We measured the input-output relationship of the number of action potentials elicited by depolarizing injections of 
increasing current intensities as our measure of membrane excitability (Fig. 6). CA1 neurons of DIV 6-8 hippocampal slices exposed to PTX (12-18 h) showed decreased membrane excitability (fewer spikes per current injected) relative to untreated controls $(p<0.01$, two-way ANOVA) (Fig. 6A, $B$, compare PTX and control).

There was relatively little spike frequency adaptation in these neurons. For example, in response to a current injection of $300 \mathrm{pA}$, average instantaneous AP frequency for the first five spike intervals ( $\mathrm{du}$ ration between successive AP peaks) was (in $\mathrm{Hz}$ ) 59, 56, 51 50, and 50. Moreover, treatment with PTX caused a decrease in firing frequency for later intervals comparable with the initial decrease $(30-37 \%$ decrease in spike frequency for each of first five spike intervals).

Cells transfected with GFP alone also displayed a drop in excitability after 12-18 h of PTX ( $p<0.01$, two-way ANOVA), comparable with that seen in neighboring untransfected cells (Fig. 6C). In contrast, neurons overexpressing the dominant interfering construct GFP-PBD failed to show a drop in membrane excitability with PTX treatment (Fig. 6A, B), indicating that Plk2 function is required for the homeostatic decline in membrane excitability during prolonged hyperactivity. As was the case for synaptic transmission (EPSC amplitude), overexpression of the dominantnegative GFP-PBD had no effect on membrane excitability in unstimulated slices (Fig. 6A, $B$, compare GFP-PBD and control).

The drop in membrane excitability was not attributable to changes in passive neuronal properties. The membrane resistance, whole-cell capacitance, and $-70 \mathrm{mV}$ holding current (reflecting the resting membrane potential) were not significantly different between untreated and PTX-treated conditions (supplemental Table S1, available at www. jneurosci.org as supplemental material). Given that passive membrane properties were unchanged, the drop in membrane excitability after PTX treatment is more likely to arise from changes in voltage-gated conductances. Consistent with this idea, analysis of AP parameters showed that PTX treatment caused a significant elevation in the AP threshold potential (supplemental Table S2, available at www.jneurosci.org as supplemental material; Fig. $6 D, E)(p<0.05$, one-way ANOVA $)$. The increase in AP threshold potential was prevented by overexpression of GFPPBD, but not by GFP, consistent with the finding that GFPPBD prevented the drop in membrane excitability (Fig. 6D,E). Our results suggest that Plk2 reduces excitability during epileptiform activity by regulating (directly or indirectly) active membrane conductances.
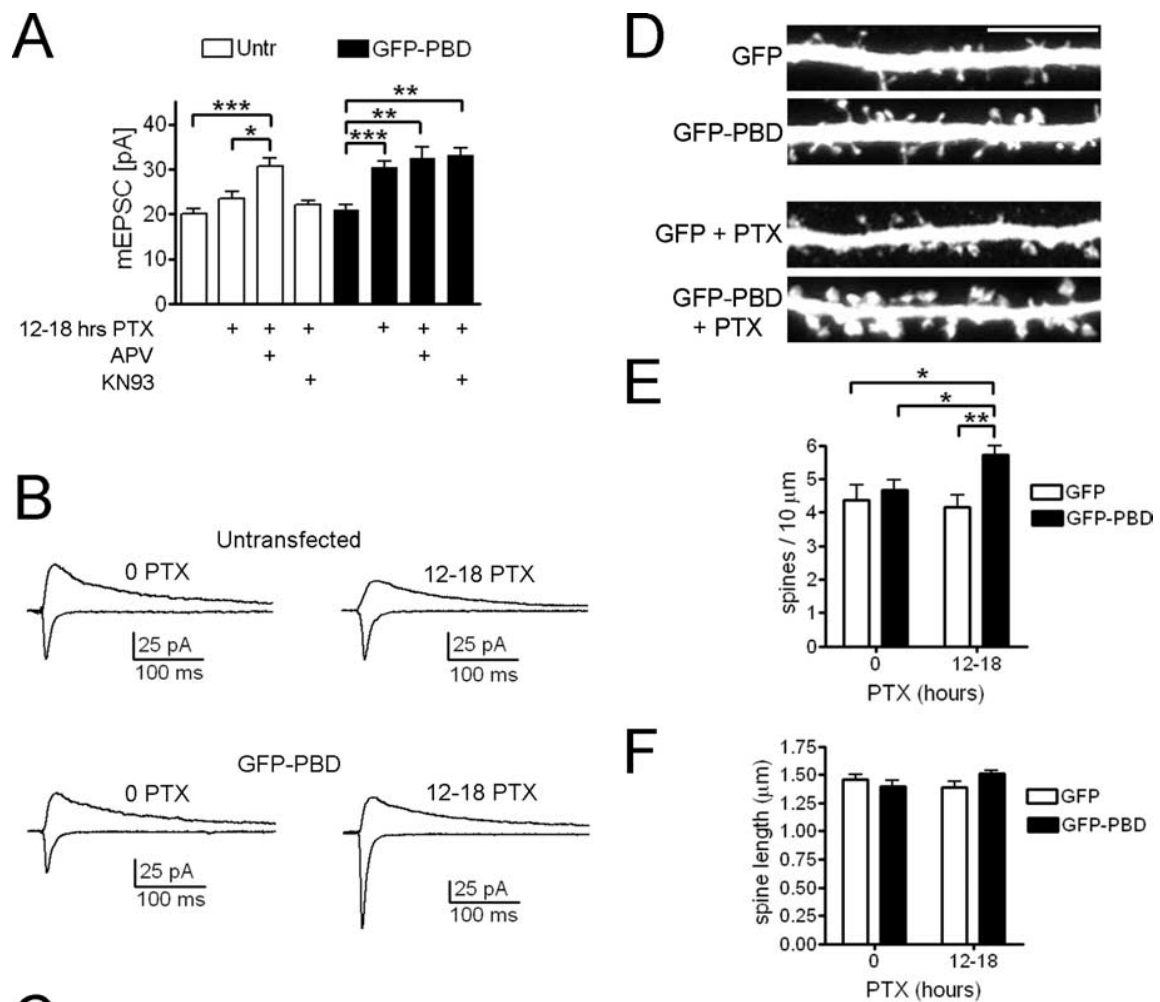

G

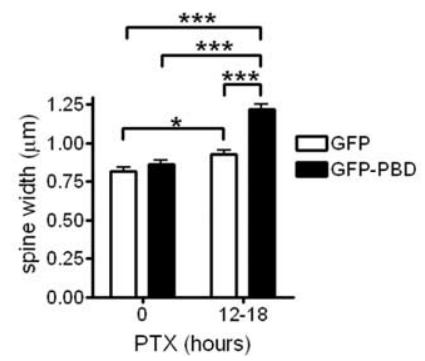

Figure 4. Characterization of epileptiform potentiation. $\boldsymbol{A}$, Effects of APV and KN93 on epileptiform potentiation. mEPSC amplitudes (mean \pm SEM) were quantitated from transfected (GFP-PBD) or untransfected (Untr) CA1 pyramidal cells either untreated or treated with PTX (100 $\mu \mathrm{m})$ for 12-18h, in combination with APV (100 $\mu \mathrm{m})$ or KN93 $(5 \mu \mathrm{m})$, as indicated. $n=5$ cells (untransfected/PTX/KN93; Untr/PTX); $n=6$ cells (GFP-PBD/PTX/KN93); $n=7-11$ cells (all other conditions). ${ }^{*} p<0.05 ;{ }^{* *} p<$ mediated EPSCs recorded from transfected (GFP-PBD) or untransfected CA1 pyramidal cells, untreated or treated with PTX (100 $\mu \mathrm{M}$ ) for 12-18 h, as indicated. C, Quantification of AMPA/NMDA ratio (mean $\pm \mathrm{SEM}$ ). $n=8$ cells per condition. ${ }^{*} p<0.05$; P7 organotypic hippocampal slice cultures (DIV 5-7) were transfected biolistically with GFP or GFP-PBD and treated with PTX (100 $\mu \mathrm{M} ; 12-18 \mathrm{~h}$ ), as indicated. Slices were fixed $\sim 24 \mathrm{~h}$ after transfection and imaged for spine morphology. Bar graphs (mean \pm 6 cells (GFP); $n=10$ cells (GFP + PTX); $n=9$ cells (GFP-PBD); $n=9$ cells (GFP-PBD + PTX). ${ }^{*} p<0.05,{ }^{* *} p<0.01,{ }^{* *} p<$ 0.001 , one-way ANOVA. D, Scale bar, $10 \mu \mathrm{m}$.

\section{Discussion}

We have shown recently that the activity-inducible protein kinase Plk2 is critical for conventional synaptic scaling in dissociated cultures of hippocampal neurons, specifically for the downregulation of synaptic strength in response to chronic PTX treatment (Seeburg et al., 2008). Here we test the role of Plk2 during epileptiform activity in hippocampal slice cultures, which is elicited by $\mathrm{GABA}_{\mathrm{A}}$ receptor blockade and often used as an in vitro model of epilepsy (Schwartzkroin and Prince, 1978; Traub and Wong, 1982; Drakew et al., 1996; Thompson et al., 1996; Karnup and Stelzer, 2001). We report that during epileptiform activity in this preparation neuronal activity is stabilized, synapses are kept within a modifiable 
A
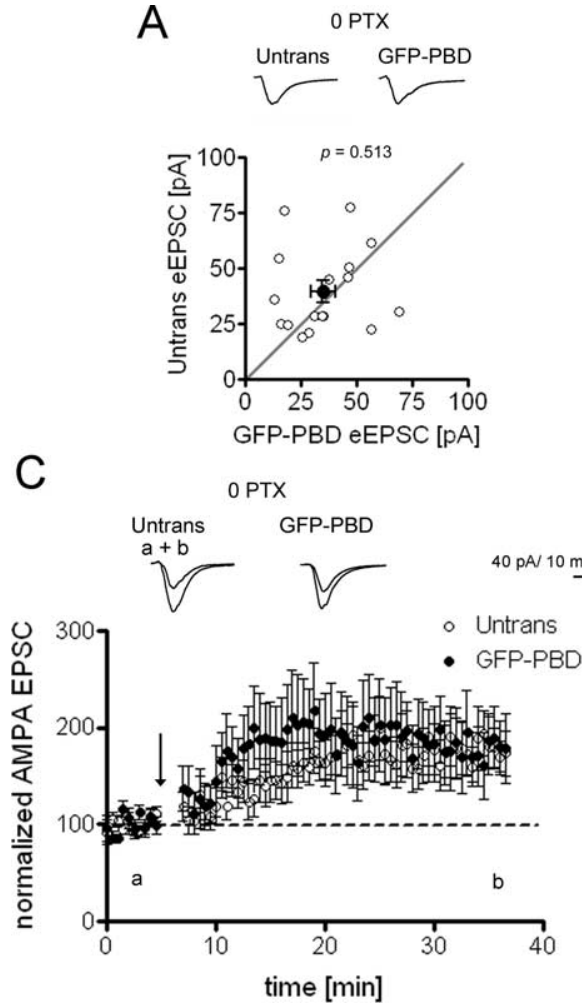

B

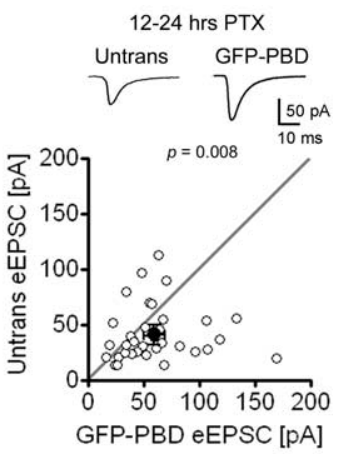

D

GFP-PBD eEPSC [pA]
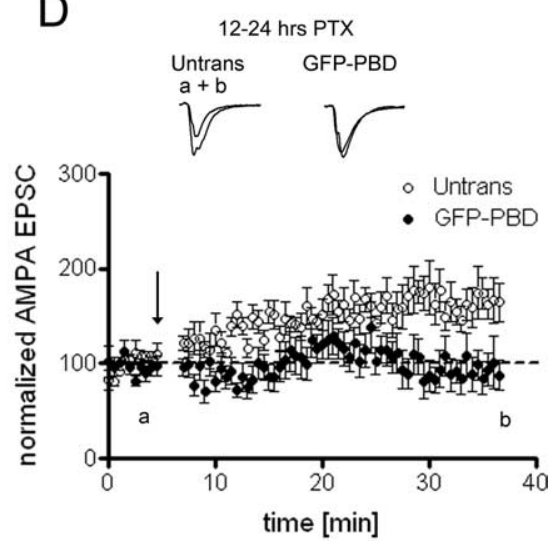

Figure 5. Plk2 function is required for LTP induction during epileptiform activity. $A, B$, Enhanced synaptic strength in GFPPBD-transfected CA1 pyramidal cells after 12-24h of PTX (100 $\mu \mathrm{m})$. Top, Sample evoked AMPA-EPSC traces are shown from pairs of transfected (GFP-PBD) and neighboring untransfected (Untrans) cells with $(\boldsymbol{B})$ or without $(\boldsymbol{A})$ prior treatment of slices with PTX for 12-24 h. Bottom, Graphs show eEPSC amplitudes plotted for each individual pair of transfected and neighboring untransfected cells (open circles). Filled circles represent the mean \pm SEM. $n=17$ cell pairs ( 0 PTX); $n=35$ cell pairs (12-24h PTX). $p$ values shown in the graph are calculated by Mann-Whitney test. $C, \mathbf{D}$, Impaired LTP in GFP-PBD-transfected CA1 pyramidal cells after 12-24h of PTX treatment. Recordings were obtained simultaneously from pairs of transfected (GFP-PBD) and untransfected (Untrans) CA1 pyramidal cells from the same slice. LTP was induced by pairing depolarization to $0 \mathrm{mV}$ with $2 \mathrm{~Hz}$ stimulation for 100 s. Top, Sample AMPAR-mediated eEPSC traces are shown from transfected and untransfected neurons without ( $($ ) or with (D) prior treatment with PTX for 12-24h. Graphs show time course of EPSCS (mean \pm SEM), normalized to baseline eEPSC amplitude before delivery of pairing protocol (arrow). Sample traces were obtained at time points indicated by a and b. $n=7-8$ cell pairs.

range, and membrane excitability is downregulated through a homeostatic mechanism requiring Plk2.

\section{Plk2-dependent homeostatic plasticity in organotypic hippocampal slice cultures}

In synaptic scaling, prolonged treatment with $\mathrm{GABA}_{\mathrm{A}}$ receptor antagonists results in a neuron-wide suppression of mEPSC amplitude, which depends on Plk2 (Seeburg et al., 2008) and is thought to represent a homeostatic response to the prolonged network hyperactivity (Turrigiano et al., 1998; Turrigiano, 2007). In contrast, we found that similar disinhibition of hippocampal slice cultures resulted in an initial upswing of synaptic strength, followed by a Plk2-dependent return toward baseline by $\sim 12-18$ $\mathrm{h}$. In both dissociated and slice culture, these responses to hyperactivity correlate with $\mathrm{Plk} 2$ induction and both require Plk2 function, suggesting that they are different manifestations of a similar homeostatic mechanism.

Homeostatic mechanisms in slice culture are manifested as the plateauing and subsequent downward shifting in the mEPSC amplitude curve that starts $\sim 6-10 \mathrm{~h}$ after PTX treatment. Our results indicate that this homeostatic response depends on Plk2, because disruption of Plk2 function by dominant-negative overexpression or RNAi led to a continued and progressive increase in synaptic strength. The difference between this increase in synap-

tic strength when Plk2 function is disrupted ("epileptiform potentiation") and the normal downward shift in mEPSC amplitude was quite large. By $12-18 \mathrm{~h}$ of epileptiform activity, the net decrease in mEPSC amplitude that could be attributed to Plk2 was from $35.89 \pm 3$ pA to $23.52 \pm$ $2.03 \mathrm{pA}$, an $\sim 34 \%$ drop in synaptic strength. Thus, Plk2-dependent plasticity mechanisms account for a significant decline in synaptic strength during epileptiform activity and thereby keep synapses close to baseline strength. Such dampening of synaptic strength would tend to stabilize neuronal activity during ongoing epileptiform activity, which is critical for any homeostatic plasticity mechanism.

One possible mechanism through which Plk2 prevents epileptiform potentiation in hippocampal slice cultures is synaptic scaling, because Plk2 is required for synaptic scaling during elevated activity in dissociated cultures (Seeburg et al., 2008). Consistent with this idea, overexpression of Plk2 by viral infection caused weakening of synapses in slice cultures undergoing long-term PTX treatment, suggesting that elevated Plk2 expression is sufficient to dampen synapses.

Another possible, and not mutually exclusive, mechanism for Plk2dependent homeostatic plasticity in slice culture involves the activity- and Plk2dependent reduction in membrane excitability. By reducing the depolarization triggered by excitatory inputs, such a drop in membrane excitability would make it more difficult for synapses to become potentiated during synchronous bursting activity: presynaptic firing would be less able to drive postsynaptic activity, resulting in less well correlated presynaptic and postsynaptic activity and thus an elevation of the synaptic modification threshold. This is analogous to what has been proposed in metaplasticity models such as the Bienenstock Cooper Munro model (Bienenstock et al., 1982; Bear, 2003 ), in which prolonged elevation in synaptic activity is predicted to result in a rightward shift in the synaptic modification threshold curve (i.e., it becomes more difficult to induce LTP), but for which molecular mechanisms are still largely unknown (Bear, 2003; Philpot et al., 2007).

According to such metaplasticity models, elevating the modification threshold during extended periods of increased neural activity keeps synapses within a dynamic range of modifiability by preventing saturation of plasticity and, thus, should have a stabilizing effect on postsynaptic activity over time (Abraham and Tate, 1997). Our data are consistent with this idea. In cells lacking Plk2 function (and showing progressive epileptiform potentiation), we were unable to elicit further potentiation through conventional LTP protocols, suggesting saturation of plasticity. In contrast, control cells in the same slice with intact Plk2 function remained modifiable by LTP. 
Potentiation in synaptic strength during epileptiform activity

When the homeostatic function of Plk2 was blocked in CA1 neurons of hippocampal slice cultures, PTX treatment resulted in escalation of synaptic strength over the course of 12-18 h. Given the high synchronicity of the bursts between areas CA3 and CA1, the epileptiform potentiation might be expected to result from Hebbian plasticity mechanisms (i.e., LTP). Surprisingly, however, antagonists of NMDARs failed to prevent the surge in synaptic strength, indicating that this unmasked form of potentiation is not attributable to conventional NMDAR-dependent LTP. Some forms of non-NMDAR-dependent LTP have been reported previously in CA1, including VDCC (voltage-dependent calcium channel)-dependent LTP (Grover and Teyler, 1990; Moosmang et al., 2005) and mGluR5/PKA-dependent LTP (Lante et al., 2006).

Overall, our results indicate that epileptiform activity elicits two forms of synaptic modification in organotypic slice cultures: a slow and progressive potentiation that occludes or saturates LTP, and a Plk2dependent dampening of membrane excitability and synaptic strength that counteracts this potentiation. In normal conditions, Plk2 is induced by elevated activity and reins in synaptic strength close to baseline, thereby maintaining the ability of synapses to undergo LTP. Insofar as mEPSC amplitude does not fall beneath baseline, this form of homeostasis that occurs during epileptiform activity in hippocampal slice cultures differs from the classical synaptic scaling seen in dispersed cultures. However, the fact that Plk2, which is important for synaptic scaling (Seeburg et al., 2008), plays a critical role in this form of plasticity, as well as in dampening of membrane excitability, strongly suggests that it is a true homeostatic response to epileptiform activity.

\section{Plk2 as endogenous antiepileptogenic or} anticonvulsive mechanism?

Epileptogenesis refers to the process by which insult(s) to the brain trigger a number of molecular, structural, and functional changes that eventually result in recurrent seizures or epilepsy (Lukasiuk et al., 2006). However, although many patients sustain brain injuries that are associated with epileptogenesis, only a small subpopulation go on to develop epilepsy. The factors that determine whether patients with brain injuries will develop epilepsy are unknown (Elliott and Lowenstein, 2004; Pitkanen et al., 2007).

One of the fundamental ways in which brain function is altered after a seizure is in gene expression (Elliott and Lowenstein, 2004). Thus, in the hope of determining key molecular alterations driving the pathogenesis of epilepsy, a number of gene expression profiling studies have attempted to compile genes that are differentially regulated after seizures or status epilepticus (Hendriksen et al., 2001; Tang et al., 2002; Becker et al., 2003;
Elliott et al., 2003; Lukasiuk et al., 2003; Gorter et al., 2006). Little is yet known about the specific functions of these seizureregulated genes. Some likely contribute to epileptogenic processes, including network reorganization and neuronal hyperexcitability (Elliott and Lowenstein, 2004; Lukasiuk et al., 2006; Crino, 2007; Pitkanen et al., 2007), but others probably constitute a compensatory or protective response to the seizure (Pitkanen et al., 2007).

Given that Plk2 is robustly induced in brain after seizures in vivo (Kauselmann et al., 1999; Newton et al., 2003), and inhibits synaptic strength and membrane excitability in hippocampal slices during epileptiform activity, we speculate that Plk2 also has antiepileptogenic properties in vivo.

\section{References}

Abegg MH, Savic N, Ehrengruber MU, McKinney RA, Gahwiler BH (2004) Epileptiform activity in rat hippocampus strengthens excitatory synapses. J Physiol (Lond) 554:439-448.

Abraham WC, Tate WP (1997) Metaplasticity: a new vista across the field of synaptic plasticity. Prog Neurobiol 52:303-323.

Bausch SB, McNamara JO (2004) Contributions of mossy fiber and CA1 pyramidal cell sprouting to dentate granule cell hyperexcitability in kainic acid-treated hippocampal slice cultures. J Neurophysiol 92:3582-3595.

Bear MF (2003) Bidirectional synaptic plasticity: from theory to reality. Philos Trans R Soc Lond B Biol Sci 358:649-655.

Becker AJ, Chen J, Zien A, Sochivko D, Normann S, Schramm J, Elger CE Wiestler OD, Blumcke I (2003) Correlated stage- and subfield- 
associated hippocampal gene expression patterns in experimental and human temporal lobe epilepsy. Eur J Neurosci 18:2792-2802.

Bienenstock EL, Cooper LN, Munro PW (1982) Theory for the development of neuron selectivity: orientation specificity and binocular interaction in visual cortex. J Neurosci 2:32-48.

Brummelkamp TR, Bernards R, Agami R (2002) A system for stable expression of short interfering RNAs in mammalian cells. Science 296:550-553.

Crino PB (2007) Gene expression, genetics, and genomics in epilepsy: some answers, more questions. Epilepsia 48 [Suppl 2]:42-50.

Davis GW (2006) Homeostatic control of neural activity: from phenomenology to molecular design. Annu Rev Neurosci 29:307-323.

Drakew A, Muller M, Gahwiler BH, Thompson SM, Frotscher M (1996) Spine loss in experimental epilepsy: quantitative light and electron microscopic analysis of intracellularly stained CA3 pyramidal cells in hippocampal slice cultures. Neuroscience 70:31-45.

Elliott RC, Lowenstein DH (2004) Gene expression profiling of seizure disorders. Neurochem Res 29:1083-1092.

Elliott RC, Miles MF, Lowenstein DH (2003) Overlapping microarray profiles of dentate gyrus gene expression during development- and epilepsyassociated neurogenesis and axon outgrowth. J Neurosci 23:2218-2227.

Gahwiler BH, Capogna M, Debanne D, McKinney RA, Thompson SM (1997) Organotypic slice cultures: a technique has come of age. Trends Neurosci 20:471-477.

Gorter JA, van Vliet EA, Aronica E, Breit T, Rauwerda H, Lopes da Silva FH, Wadman WJ (2006) Potential new antiepileptogenic targets indicated by microarray analysis in a rat model for temporal lobe epilepsy. J Neurosci 26:11083-11110.

Grover LM, Teyler TJ (1990) Two components of long-term potentiation induced by different patterns of afferent activation. Nature 347:477-479.

Hendriksen H, Datson NA, Ghijsen WE, van Vliet EA, da Silva FH, Gorter JA, Vreugdenhil E (2001) Altered hippocampal gene expression prior to the onset of spontaneous seizures in the rat post-status epilepticus model. Eur J Neurosci 14:1475-1484.

Holopainen IE (2005) Organotypic hippocampal slice cultures: a model system to study basic cellular and molecular mechanisms of neuronal cell death, neuroprotection, and synaptic plasticity. Neurochem Res 30:1521-1528.

Jiang N, Wang X, Jhanwar-Uniyal M, Darzynkiewicz Z, Dai W (2006) Polo box domain of Plk3 functions as a centrosome localization signal, overexpression of which causes mitotic arrest, cytokinesis defects, and apoptosis. J Biol Chem 281:10577-10582.

Karmarkar UR, Buonomano DV (2006) Different forms of homeostatic plasticity are engaged with distinct temporal profiles. Eur J Neurosci 23:1575-1584.

Karnup S, Stelzer A (2001) Seizure-like activity in the disinhibited CA1 minislice of adult guinea-pigs. J Physiol (Lond) 532:713-730.

Kauselmann G, Weiler M, Wulff P, Jessberger S, Konietzko U, Scafidi J, Staubli U, Bereiter-Hahn J, Strebhardt K, Kuhl D (1999) The polo-like protein kinases Fnk and Snk associate with a $\mathrm{Ca}(2+)$ - and integrinbinding protein and are regulated dynamically with synaptic plasticity. EMBO J 18:5528-5539.

Kopec CD, Li B, Wei W, Boehm J, Malinow R (2006) Glutamate receptor exocytosis and spine enlargement during chemically induced long-term potentiation. J Neurosci 26:2000-2009.

Lante F, de Jesus Ferreira MC, Guiramand J, Recasens M, Vignes M (2006) Low-frequency stimulation induces a new form of LTP, metabotropic glutamate (mGlu5) receptor- and PKA-dependent, in the CA1 area of the rat hippocampus. Hippocampus 16:345-360.

Lowery DM, Mohammad DH, Elia AE, Yaffe MB (2004) The Polo-box domain: a molecular integrator of mitotic kinase cascades and Polo-like kinase function. Cell Cycle 3:128-131.

Lukasiuk K, Kontula L, Pitkanen A (2003) cDNA profiling of epileptogenesis in the rat brain. Eur J Neurosci 17:271-279.

Lukasiuk K, Dabrowski M, Adach A, Pitkanen A (2006) Epileptogenesisrelated genes revisited. Prog Brain Res 158:223-241.

Malinow R, Schulman H, Tsien RW (1989) Inhibition of postsynaptic PKC or CaMKII blocks induction but not expression of LTP. Science 245:862-866.
Matsuzaki M, Honkura N, Ellis-Davies GC, Kasai H (2004) Structural basis of long-term potentiation in single dendritic spines. Nature 429:761-766.

Moosmang S, Haider N, Klugbauer N, Adelsberger H, Langwieser N, Muller J, Stiess M, Marais E, Schulla V, Lacinova L, Goebbels S, Nave KA, Storm DR, Hofmann F, Kleppisch T (2005) Role of hippocampal Cav1.2 $\mathrm{Ca}^{2+}$ channels in NMDA receptor-independent synaptic plasticity and spatial memory. J Neurosci 25:9883-9892.

Newton SS, Collier EF, Hunsberger J, Adams D, Terwilliger R, Selvanayagam E, Duman RS (2003) Gene profile of electroconvulsive seizures: induction of neurotrophic and angiogenic factors. J Neurosci 23:10841-10851.

Okamoto K, Nagai T, Miyawaki A, Hayashi Y (2004) Rapid and persistent modulation of actin dynamics regulates postsynaptic reorganization underlying bidirectional plasticity. Nat Neurosci 7:1104-1112.

Pak DT, Sheng M (2003) Targeted protein degradation and synapse remodeling by an inducible protein kinase. Science 302:1368-1373.

Philpot BD, Cho KK, Bear MF (2007) Obligatory role of NR2A for metaplasticity in visual cortex. Neuron 53:495-502.

Pitkanen A, Kharatishvili I, Karhunen H, Lukasiuk K, Immonen R, Nairismagi J, Grohn O, Nissinen J (2007) Epileptogenesis in experimental models. Epilepsia 48 [Suppl 2]:13-20.

Rutherford LC, Nelson SB, Turrigiano GG (1998) BDNF has opposite effects on the quantal amplitude of pyramidal neuron and interneuron excitatory synapses. Neuron 21:521-530.

Sala C, Futai K, Yamamoto K, Worley PF, Hayashi Y, Sheng M (2003) Inhibition of dendritic spine morphogenesis and synaptic transmission by activity-inducible protein Homerla. J Neurosci 23:6327-6337.

Schwartzkroin PA, Prince DA (1978) Cellular and field potential properties of epileptogenic hippocampal slices. Brain Res 147:117-130.

Seeburg DP, Feliu M, Gaiottino J, Sheng M (2008) Critical role of CDK5 and Polo-like kinase 2 in homeostatic synaptic plasticity during elevated activity. Neuron 58:571-583.

Seong YS, Kamijo K, Lee JS, Fernandez E, Kuriyama R, Miki T, Lee KS (2002) A spindle checkpoint arrest and a cytokinesis failure by the dominantnegative polo-box domain of Plk1 in U-2 OS cells. J Biol Chem 277:32282-32293.

Sheng M, Hoogenraad CC (2007) The postsynaptic architecture of excitatory synapses: a more quantitative view. Annu Rev Biochem 76:823-847.

Shepherd JD, Rumbaugh G, Wu J, Chowdhury S, Plath N, Kuhl D, Huganir RL, Worley PF (2006) Arc/Arg3.1 mediates homeostatic synaptic scaling of AMPA receptors. Neuron 52:475-484.

Silva AJ, Stevens CF, Tonegawa S, Wang Y (1992) Deficient hippocampal long-term potentiation in alpha-calcium-calmodulin kinase II mutant mice. Science 257:201-206.

Song S, Lee KS (2001) A novel function of Saccharomyces cerevisiae CDC5 in cytokinesis. J Cell Biol 152:451-469.

Song S, Grenfell TZ, Garfield S, Erikson RL, Lee KS (2000) Essential function of the polo box of $\mathrm{Cdc} 5$ in subcellular localization and induction of cytokinetic structures. Mol Cell Biol 20:286-298.

Stellwagen D, Malenka RC (2006) Synaptic scaling mediated by glial TNFalpha. Nature 440:1054-1059.

Stoppini L, Buchs PA, Muller D (1991) A simple method for organotypic cultures of nervous tissue. J Neurosci Methods 37:173-182.

Tang Y, Lu A, Aronow BJ, Wagner KR, Sharp FR (2002) Genomic responses of the brain to ischemic stroke, intracerebral haemorrhage, kainate seizures, hypoglycemia, and hypoxia. Eur J Neurosci 15:1937-1952.

Thompson SM, Fortunato C, McKinney RA, Muller M, Gahwiler BH (1996) Mechanisms underlying the neuropathological consequences of epileptic activity in the rat hippocampus in vitro. J Comp Neurol 372:515-528.

Traub RD, Wong RK (1982) Cellular mechanism of neuronal synchronization in epilepsy. Science 216:745-747.

Turrigiano G (2007) Homeostatic signaling: the positive side of negative feedback. Curr Opin Neurobiol 17:318-324.

Turrigiano GG, Leslie KR, Desai NS, Rutherford LC, Nelson SB (1998) Activity-dependent scaling of quantal amplitude in neocortical neurons. Nature 391:892-896.

van de Weerdt BC, Medema RH (2006) Polo-like kinases: a team in control of the division. Cell Cycle 5:853-864.

Zha XM, Green SH, Dailey ME (2005) Regulation of hippocampal synapse remodeling by epileptiform activity. Mol Cell Neurosci 29:494-506. 